\title{
Hypersensibilität bei Tieren phytotherapeutisch behandeln
}

\author{
Yvonne Thoonsen
}

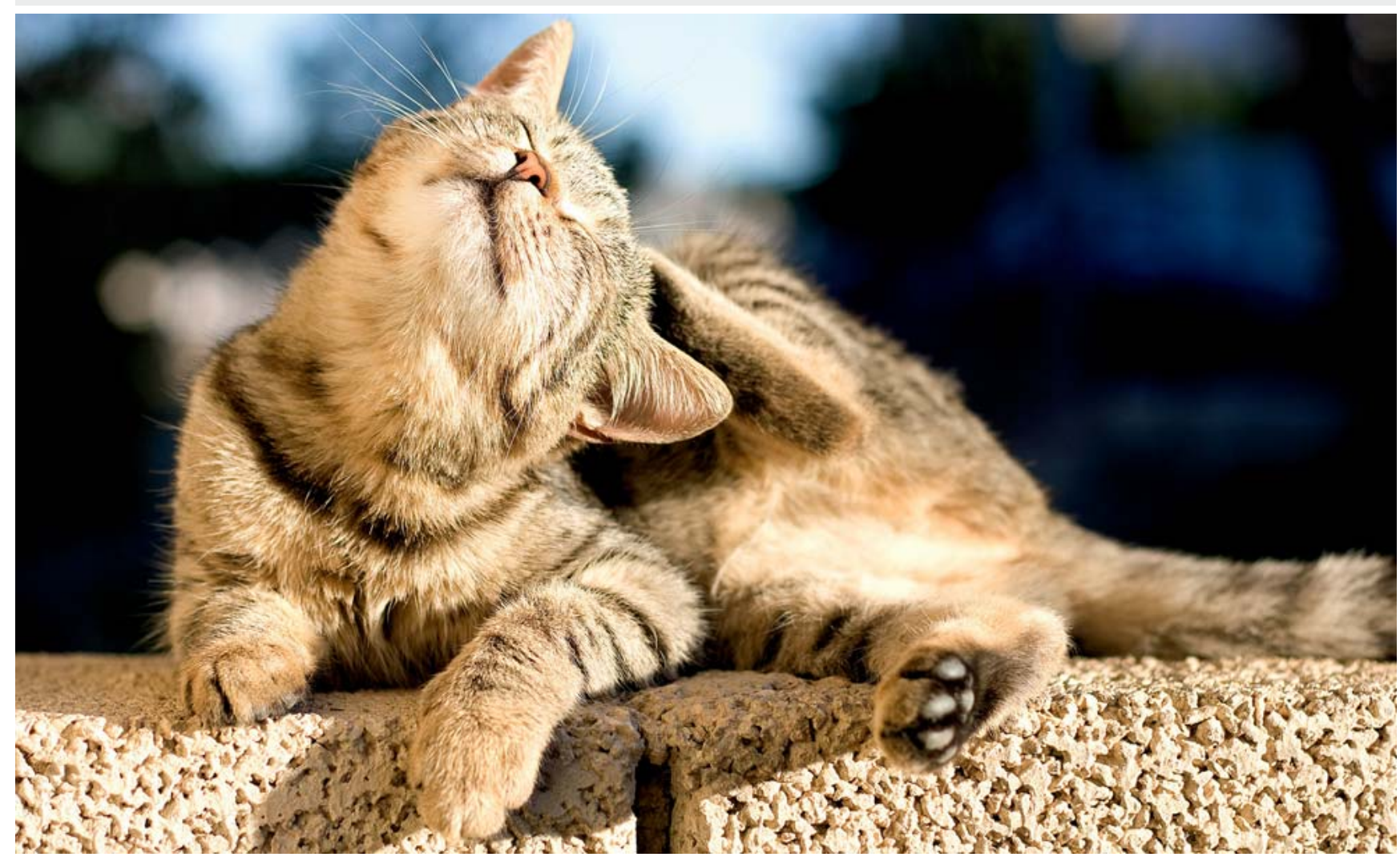

Juckreiz ist ein häufiges Symptom bei allergischen Reaktionen und Überempfindlichkeitsreaktionen der Haut. @ jelena990 - stock.adobe.com

Viele Symptome bei Hautpatienten sind die Folge einer Allergie oder Unverträglichkeit. Die Ätiologie ist multifaktoriell. Phytotherapeutische Therapieansätze können dabei helfen, die Hautbarriere wiederherzustellen sowie Pruritus, Entzündungen und Superinfektionen der Haut zu therapieren.

\section{Einleitung}

Rund $50 \%$ der uns vorgestellten Hautpatienten zeigen Symptome infolge einer allergischen Reaktion bzw. einer Unverträglichkeitsreaktion auf Futterkomponenten, Umweltallergene, oder aber auf chemisch-synthetische Stoffe. Die Patienten werden mit einer mehr oder weniger lokal begrenzt entzündeten, teils infizierten, v. a. aber juckenden Haut vorgestellt. Nur in wenigen der uns vorgestellten Fälle sind zur Therapie Kortison und Antibiotika erforderlich. Die Pflanzenheilkunde verfügt über eine Reihe unterstützender Mittel zur Behandlung allergischer und nicht-allergischer Überempfindlichkeiten sowohl in
Bezug auf akuten Juckreiz, Pruritus und Hautinfektionen als auch zur Prophylaxe und für das Langzeitmanagement.

\section{Ätiologie und Pathogenese}

Die Ätiologie von v. a. allergischen Überempfindlichkeitsreaktionen ist multifaktoriell. Gendefekte mit Wirkung auf die Hautfunktionen gibt es in Form z. B. der Filaggrin-Mutation, die zum Strukturverlust der Epidermis führt sowie unzureichender Ceramid-Konzentrationen im Stratum corneum, die zu Wasserverlusten der Haut und damit zu einer trockenen, infektanfälligen Haut füh- 


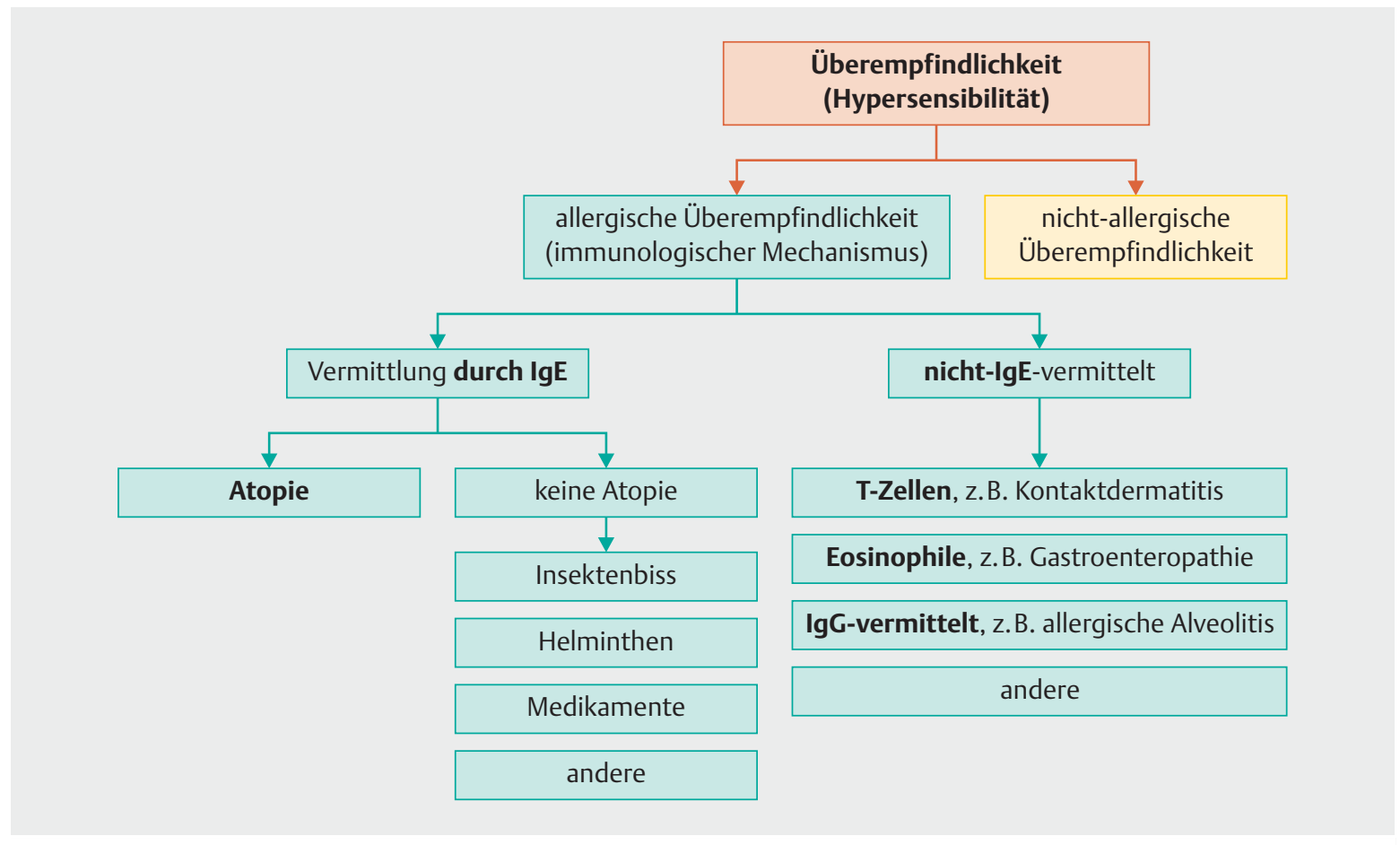

- Abb. 1 Klassifikation der Überempfindlichkeitsreaktionen nach der EAACI (European Academy of Allergy and Clinical Immunology).

ren. Die zu den Ceramiden gehörende Sphingosine dienen der gesunden Haut aufgrund ihrer antibakteriellen Wirkung als Infektionsschutz. Sphingosin-Mangel reduziert demnach die Infektabwehr der Haut. Zudem können z. B. auch Typ-1-Allergien (lgE-vermittelt) auftreten [8].

Störungen des Immunsystems, die möglicherweise in einer nicht oder nur unzureichend erworbenen oralen Toleranz im Welpen-/Junghundealter begründet sein können, führen möglicherweise bereits früh z.B. zu einem gestörten Verhältnis der Th1- und Th2-Immunantwort. Zudem kann die Mastzellaktivität durch IgE-Bindung pathologisch erhöht sein. Und nicht selten findet sich eine erhöhte Anzahl eosinophiler Granulozyten in der geschädigten Haut.

Bei Katzen und auch Hunden liegt der atopischen Dermatitis scheinbar überwiegend eine Typ-I-Hypersensibilitätsreaktion zugrunde. Allergene binden an epidermale Langerhans-Zellen und werden so allergen-spezifischen T-Lymphozyten präsentiert, was zu einer vermehrten Bildung von IgE und Mastzellen führt. Durch die Verknüpfung von auf Mastzellen gebundenem IgE kommt es zur Ausschüttung von Entzündungsmediatoren wie Histamin, Heparin, proteolytischen Enzymen, Prostaglandinen und Leukotrienen und zur akuten allergischen Reaktion [4].
Allergene dringen dabei nicht nur direkt über die Haut ein. Auch über den Atmungstrakt oder über den Verdauungstrakt eingedrungene Allergene können zu Überempfindlichkeitsreaktionen mit Hauterscheinungen führen.

\section{Grundsätzliche Therapieansätze}

Die nachfolgend genannten Ansätze kommen meist zusätzlich zu den üblichen schulmedizinischen Therapeutika (Glukokortikoide, Antihistaminika etc.) zum Einsatz.

\section{THERAPIEANSÄTZE}

- Allergenvermeidung - sofern möglich

- Kontrolle/Prophylaxe von sogenannten „Flarefaktoren“

- Allergenspezifische Immuntherapie (ASIT)

- Reduktion der Stoffwechselbelastung des Organismus und Unterstützung der Ausscheidungskapazitäten

- Förderung der Darmgesundheit - Wiederherstellung, Erhöhung der mikrobiellen Diversität im Darm

- Verbesserung der kutanen Barrierefunktion

- Therapie von Pruritus, Entzündungsreaktionen und Wunden sowie von Dermatophytosen und bakteriellen Infektionen 


\section{Allergenvermeidung}

Die Vermeidung des Allergenkontaktes bei Allergikern ist bei Futtermitteln als Auslöser noch am ehesten möglich. Umweltallergene zu meiden ist hingegen kaum möglich, da man die sensibilisierenden Allergene meist nicht identifizieren oder nicht eliminieren kann.

\section{Kontrolle/Prophylaxe von „Flarefaktoren“}

Als „Flarefaktoren“ werden z. B. Flöhe, Milben, Bakterien und auch Hautpilze bezeichnet. Die überempfindliche Haut ist zusätzlich anfälliger für Infektionen. Diese gilt es insbesondere bei Allergikern zu eliminieren, um die überforderte Haut/das überschießend reagierende Immunsystem nicht noch zusätzlich zu belasten.

\section{Allergenspezifische Immuntherapie (ASIT) = Desensibilisierung)}

Die Erfolgsrate bei dieser Therapie liegt bei Katzen bei ca. $70 \%$. Je jünger die Tiere zu Beginn der Therapie sind, desto höher sind die Erfolgschancen und desto zügiger stellen sich in der Regel die Erfolge ein. Grundsätzlich kann es aber 8-12 Monate dauern, bis sich Erfolge abzeichnen. Zumeist ist die ASIT aber nicht allein ausreichend. Es werden immer wieder andere unterstützende Therapien erforderlich [4]. Bei Hunden ist es sehr ähnlich.

\section{Stoffwechselbelastung reduzieren und Ausscheidungskapazitäten unterstützen}

Stoffwechselbelastungen durch unverträgliche Futtermittel, metabolische Störungen, Medikationen, Toxinbelastungen etc. gilt es möglichst zu vermeiden oder aber die Ausscheidung von belastenden Stoffwechselprodukten zu fördern. Hier liegt ein besonderes Augenmerk auf der Unterstützung der Entgiftungs-/Ausscheidungsorgane wie Leber, Nieren, Darm, Lunge und auch Haut. Die Phytotherapie bietet eine Vielzahl potenter Drogen [1].

\section{Förderung der Darmgesundheit}

Symbiotische Darmbakterien beeinflussen das Gleichgewicht des Immunsystems. Sie blockieren indirekt auch jene Immunzellen, die für das Auslösen von Allergien verantwortlich sind. Die Besiedlung des Darms beginnt bei der Geburt, wobei vaginale und intestinale Bakterien der Mutter als Inokulum dienen. Der tatsächliche Einfluss der Darmflora ist nur sehr schwer abzuschätzen. In Studien korrelierte jedoch eine erhöhte mikrobielle Diversität in der Darmflora (Mausmodell) als auch in der Umwelt (Bauernhofkinder) mit einem geringeren Risiko für allergische Erkrankungen. Dieses Prinzip ist auch für Tiere anzunehmen. Die Wiederherstellung bzw. Erhöhung der mikrobiellen Diversität im Darm ist anzustreben. Auch hier helfen insbesondere Pflanzen, das Darmmilieu zu verbessern.

\section{Schwellenwertprinzip}

Man geht heute davon aus, dass prädisponierte Tiere eine Mehrzahl an Überempfindlichkeiten gleichzeitig zeigen. Jede einzelne Überempfindlichkeit für sich muss nicht zwangsläufig auch zu offensichtlichen klinischen Auffälligkeiten führen. Oft führt die Kombination mehrerer gleichzeitig belasteter Systeme zu den klassischen Symptomen [3].

\section{Einsatz von Heilpflanzen}

Wie helfen uns Heilpflanzen, Linderung zu erreichen?

Heilpflanzen helfen die Hautbarriere zu stärken und das Immunsystem zu modulieren. Sie helfen außerdem, quälenden Juckreiz zu lindern, eine aus den Fugen geratene Hautflora zu stabilisieren. Und sie verbessern und beschleunigen zuverlässig die Wundheilung. Im Bereich der Kontrolle der Flarefaktoren können sie ebenfalls hilfreich sein (s.a. Zeitschrift für Ganzheitliche Tiermedizin 2018; 32(03): 96-104, Vom Umgang mit Parasiten, doi:10.1055/a-0634-1981). Außerdem stärken und unterstützen sie die Funktion der Stoffwechsel-/u. Ausscheidungsorgane.

Die Therapie von Pruritus und Entzündungsreaktionen, die Verbesserung der kutanen Barrierefunktion, die Immunmodulation und die symptomatische Therapie der Überbesiedlung mit Bakterien und Hefen bzw. Hautpilzen sowie von Wunden sind prädisponierte Einsatzgebiete der Phytotherapie.

\section{Verbesserung der kutanen Barrierefunktion}

Zur Verbesserung der kutanen Barrierefunktion können folgende Bausteine zum Einsatz kommen:

1. Essentielle Fettsäuren (Omega-3-FS und Omega-6-FS)

2. Ceramide/Sphingosine

3. Avocadin ${ }^{\circledR}$

\section{Essentielle Fettsäuren}

Wirkprinzipien und Kerngedanken zur unterstützenden Therapie der Haut:

1. Antiinflammatorische und juckreizlindernde Wirkung durch Änderung der Bildung von Entzündungsmediatoren

2. Immunmodulatorische Wirkung durch Hemmung von Zellaktivierung und Zytokin-Sekretion

3. Wiederherstellung und Aufrechterhaltung der epidermalen Hautbarriere

4. Pflege und Beruhigung der läsionalen Haut 
\ Tab. 1 Lieferanten für wertvolle essentielle Omega-3- und Omega-6-Fettsäuren (FS).

\begin{tabular}{|c|c|c|c|}
\hline Heilpflanze & Omega-3-FS & Omega-6-FS & Wirkung \\
\hline $\begin{array}{l}\text { Lein } \\
\text { Linum usitatissimum L. (Lini oleum } \\
\text { virginale = Leinöl) }\end{array}$ & $58 \%$ $\alpha$-Linolensäure & $15 \%$ Linolsäure & $\begin{array}{l}\text { " antiinflammatorisch } \\
\text { - immunmodulierend } \\
\text { " antioxidativ } \\
\text { " Zellmembranen stärkend } \\
\text { " Zellneubildung anregend und Stärkung } \\
\text { der Hornhautbarriere }\end{array}$ \\
\hline $\begin{array}{l}\text { Borretsch } \\
\text { Borago officinalis } L \text {. (Boraginis offi- } \\
\text { cinalis oleum raffinatum = raf- } \\
\text { finiertes Borretschsamenöl) }\end{array}$ & & $\begin{array}{l}\text { 35-38\% Linolsäure } \\
20-25 \% \text { y-Linolensäure }\end{array}$ & $\begin{array}{l}\text { - antiphlogistisch } \\
\text { " immunmodulierend } \\
\text { - fördern ausgewogene Zusammenset- } \\
\text { zung des Lipidfilms der Hautoberfläche }\end{array}$ \\
\hline $\begin{array}{l}\text { Nachtkerze } \\
\text { Oenothera biennis L. (Oenothera } \\
\text { oleum raffinatum = raffiniertes } \\
\text { Nachtkerzenöl) }\end{array}$ & & $\begin{array}{l}\text { 67\% Linolsäure } \\
8-14 \% \text { y-Linolensäure }\end{array}$ & wie Borretsch \\
\hline $\begin{array}{l}\text { Hanf } \\
\text { Cannabis sativa L. (Oleum canna- } \\
\text { bis = Hanföl) }\end{array}$ & $17 \% \alpha$-Linolensäure & 54\% Linolsäure & $\begin{array}{l}\text { - entzündungshemmend } \\
\text { - immunmodulierend } \\
\text { " antioxidativ } \\
\text { " Zellmembran stärkend } \\
\text { - Zellneubildung anregend und Stärkung } \\
\text { der Hornhautbarriere }\end{array}$ \\
\hline
\end{tabular}

\section{DOSIERUNGEN}

Leinöl und Hanföl: 1-4 EL/Tier/Tag

Borretsch Öl und Nachtkerzenöl: 60-80 mg/Tier

10 kg/Tag bzw. 96-128 mg/Tier20 kg/Tag über

mind. 4-12 Wochen, ggf. dauerhaft

$[1,2,7]$

Auch Walnuss, Soja und Raps enthalten größere Mengen an $\alpha$-Linolensäure (ALA). ALA wird im Körper durch die ס-6-Desaturase zu Eicosapentaensäure (EPA) umgewandelt, während das gleiche Enzym Linolsäure (LA) zu Dihomo-y-Linolensäure (DGLA) und Arachidonsäure (AA) umwandelt. Aus DGLA und EPA werden entzündungshemmende Eicosanoide der Serie 1 und 3 und aus der gleichzeitig gebildeten AA proinflammatorische Serie-2Eicosanoide. ALA zieht die Enzymaktivität auf sich, die sonst für die Produktion der entzündungsfördernden AA zur Verfügung stehen würde. EPA wird u.a. auch weiter zu Docosahexaensäure (DHA) verstoffwechselt. Den höchsten Gehalt an „direkten“ EPA und DHA findet man in fettem Meeresfisch.

Die DGLA dient der Synthese der Serie-1- und Serie-2Eicosanoide. Die Kombination mit ALA hemmt die Konversion in die AA und damit eine Verschiebung der Umwandlung von DGLA in Richtung entzündungshemmender Serie-1- Eicosanoide [8].
Die Behandlung der caninen atopischen Dermatitis mit ungesättigten Fettsäuren hat in einer randomisierten, doppelt-verblindeten, plazebo-kontrollierten Multizenterstudie einen signifikanten Steroid-einsparenden Effekt zum Ergebnis gehabt. Auch Katzen profitieren erheblich von einer unterstützenden Therapie mit ungesättigten Fettsäuren. Insbesondere bei Hund und Mensch kann durch die orale und lokale Fettsäurensupplementierung eine erfolgreiche Wiederherstellung der Barrierefunktion der Haut und eine Verbesserung des klinischen Zustandes erreicht werden. Außerdem wirken sich die essentiellen Fettsäuren antiinflammatorisch und immunmodulierend auf v.a. die atopische Haut bzw. insgesamt aus, wodurch entzündliche Symptome und Juckreiz deutlich gemildert werden können [8].

\section{Ceramide/Sphingosine}

Ceramide sind mit bis zu ca. $18 \%$ unter anderem Bestandteil der Hornschicht der Haut (Stratum corneum). Dort bilden sie aufgrund ihrer amphiphilen Struktur Doppellipidschichten. Diese dienen als Schutzbarriere vor Austrocknung und dem Eindringen von Fremdstoffen. Ceramid I ist strukturell ein Ester der Linolsäure. Eine unzureichende Aufnahme von Linolsäure führt zu einer ungenügenden Ceramid I Bildung mit der Folge einer trockenen, oft schuppigen Haut und einer gestörten Hautbarriere $[11,12]$.

\section{Avocadin $^{\circledR}$}

Avocadin besteht aus raffiniertem Avocado-Öl und ca. $25 \%$ unverseifbaren Bestandteilen, welche ca. $80-85 \%$ 

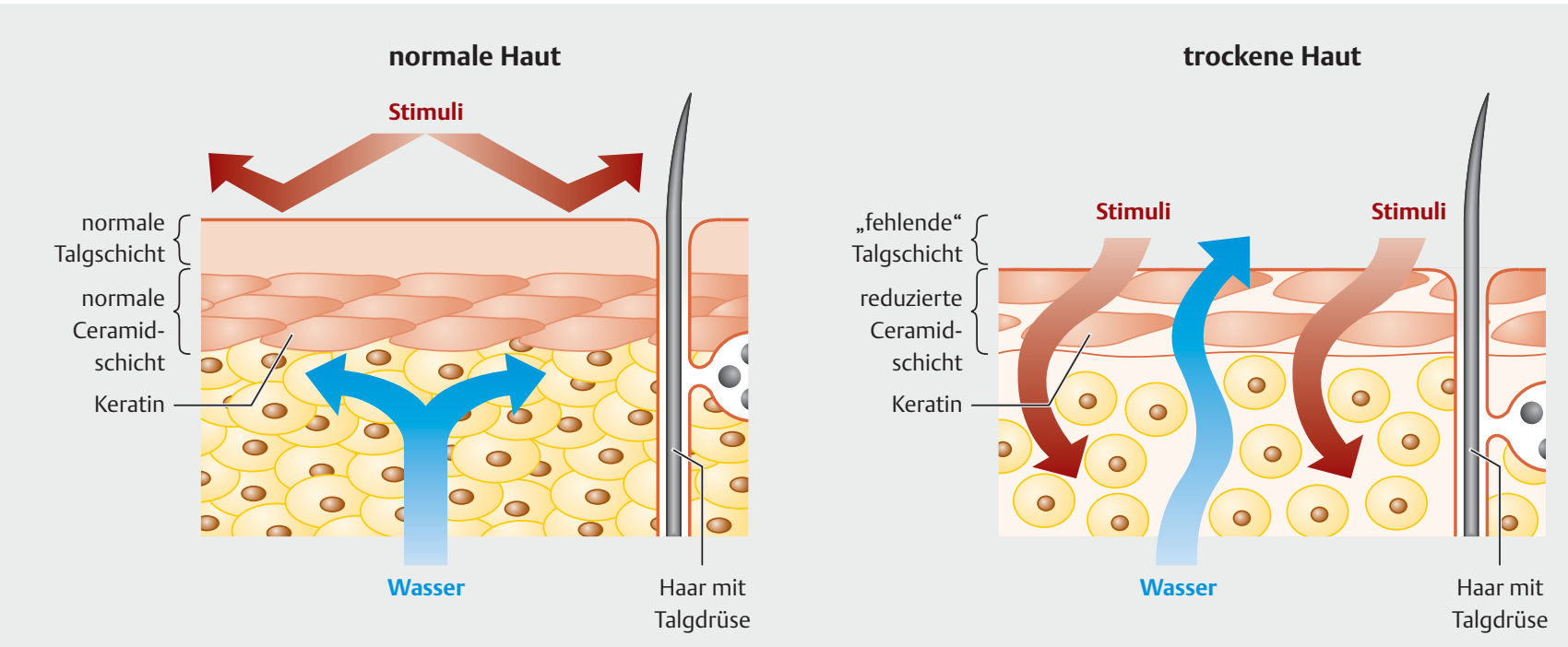

- Abb. 2 Die Ceramidschicht bei normaler und trockener Haut im Vergleich. @ Thoonsen

Sterole (Sitosterol, Stigmasterol, Campesterol und Brassicosterol) enthalten. Avocadin ${ }^{\circledR}$ enthält in hochkonzentrierter Form hautverwandte Lipide. Sie kommen natürlicherweise im Hydro-Lipid-Film und der Hornschicht unserer Haut vor. Phytosterole, die den hauteigenen Cholesterolen entsprechen, sorgen dafür, dass die Verdunstung von Wasser aus der Haut verhindert wird. Dies führt zur Elastizitätssteigerung der Haut. Es wirkt außerdem regenerierend auf eine gestörte Barrierefunktion der Haut und hilft, entzündliche Prozesse zu mildern [10].

\section{Bitterstoffe und ihre Bedeutung für die Hautbarrierefunktion}

Bitterstoffe sind in der Phytotherapie für ihren Einsatz insbesondere bei Magen-Darm-Problemen und zur Unterstützung der Leber und der Galle bekannt. Dass Bitterstoffe sich auch durch hautregenerierende und barrierestärkende Wirkungen auszeichnen, ist relativ neu. Amarogentin aus dem Gelben Enzian und Salicin aus der Weidenrinde fördern die Bildung von Proteinen, die für den Aufbau der Hautbarriere wichtig sind (z.B. Keratin und Filaggrin). Die Bitterstoffe des Gelben Enzians regen überdies die Synthese von Hautlipiden in Keratinozyten an (Ceramid III), die für die Ausbildung der Hautbarriere von Bedeutung sind. Belegt ist, dass ein Extrakt aus dem Gelben Enzian zu einer signifikanten Erhöhung der Lipidsynthese führt. Dies macht diesen Extrakt auch besonders für Atopiker interessant. Bitterstoffe haben zudem eine immunmodulierende und eine nachweislich entzündungshemmende Wirkung. Aufgrund der Art des Zustandekommens dieser Wirkung können auch TNF- $\alpha$ und Histamin-induzierte entzündliche Hauterkrankungen wie der chronischen atopischen Dermatitis und der Urtikaria von z. B. dem Bitterstoff Amarogentin profitieren [9].

Zubereitungen (Tee, Dekokt, Salben etc.) und Art der Anwendung, sofern nicht auf kommerzielle Produkte zurückgegriffen werden soll, können der Literaturstelle [1] entnommen werden. Für Interessierte findet sich hier eine Fülle weiterer Pflanzen mit nahezu allen Informationen dazu, die zum gegenwärtigen Zeitpunkt zur Verfügung stehen. Hier werden auch die ätherischen Öle und ihr Einsatz bei Hauterkrankungen ausführlich beschrieben.

\section{Therapie von Dermatophytosen}

Zur Behandlung von Dermatophytosen eignet sich z. B. das Johanniskraut (Hypericum perforatum L.), die Große Kapuzinerkresse (Tropaoelum majus L.), der Salbei (Salvia officinalis) und auch das Zitronengras (Cymbopogon citratus) [1]. Zudem verspricht ein Blick in das Reich der Pilze interessante Therapieansätze bei Mykosen und bakteriellen Infektionen der Haut.

\section{Pythium oligandrum (P. oligandrum)}

Ein sehr interessanter Pilz, der durchaus einer eingehenderen Betrachtung lohnt. P. oligandrum gehört zur Ordnung der Oomycetes und der Familie der Phytiaceae und hat ein sehr großes Wirtsspektrum. Pythium ist ein sogenannter Eipilz oder auch Scheinpilz genannt. Eipilze sind eigentlich näher mit Algen verwandt als mit den echten Pilzen. P. oligandrum ist ein Mikromycete mit mykoparasitischen Eigenschaften, der in der Landwirtschaft zur Bekämpfung von Pilzinfektionen an Pflanzen eingesetzt wird. Für die Behandlung von Dermatophytosen wurden auch Rezepturen mit Pythium oligandrum entwickelt, 
Tab. 2 Therapie von Pruritus, Entzündungsreaktionen und Wunden.

\begin{tabular}{|c|c|c|}
\hline Heilpflanze & Wirkung auf die Haut & Indikation \\
\hline $\begin{array}{l}\text { Ackerstiefmütterchen } \\
\text { Viola tricolor L., Viola herba cum flore }\end{array}$ & $\begin{array}{l}\text { Innerliche und äußerliche Anwendung: } \\
\text { " antiphlogistisch } \\
\text { " keratolytisch } \\
\text { " antimikrobiell } \\
\text { " antineuralgisch }\end{array}$ & $\begin{array}{l}\text { bei leicht seborrhoischer Haut sowie bei Ekzemen } \\
\text { und Impetigo }\end{array}$ \\
\hline $\begin{array}{l}\text { Ballonrebenkraut } \\
\text { Cardiospermum halicacabum L., Cardio- } \\
\text { spermi herba }\end{array}$ & $\begin{array}{l}\text { Äußerliche Anwendung: } \\
\text { " antiphlogistisch } \\
\text { " juckreizlindernd } \\
\text { - feuchtigkeitsspendend }\end{array}$ & $\begin{array}{l}\text { " Dermatitis } \\
\text { " Pruritus } \\
\text { " Neurodermitis } \\
\text { - Allergisches Kontaktekzem } \\
\text { " Seborrhoisches, mikrobielles, Exsikkations- } \\
\text { ekzem } \\
\text { " Insektenstiche }\end{array}$ \\
\hline $\begin{array}{l}\text { Beinwell } \\
\text { Symphytum officinale L., Symphyti radix/ } \\
\text { herba/folium }\end{array}$ & $\begin{array}{l}\text { Äußerliche Anwendung: } \\
\text { - antiphlogistisch } \\
\text { - analgetisch } \\
\text { - antiexsudativ } \\
\text { - gewebsregulierend } \\
\text { - keimhemmend } \\
\text { - Förderung Wundheilung } \\
\text { - Förderung Granulation } \\
\text { - reizlindernd }\end{array}$ & $\begin{array}{l}\text { - Nagelbettentzündung } \\
\text { " Furunkel }\end{array}$ \\
\hline $\begin{array}{l}\text { Birke } \\
\text { Betula pendula u. Betula pubescens } L \text {., } \\
\text { Betula cortex }\end{array}$ & $\begin{array}{l}\text { Äußerliche Anwendung: } \\
\text { " antitumoral } \\
\text { " antioxidativ } \\
\text { " wundheilungsfördernd } \\
\text { " antimikrobiell } \\
\text { " entzündungshemmend } \\
\text { " Hautbarriere stärkend } \\
\text { " regenerativ } \\
\text { - juckreizlindernd }\end{array}$ & $\begin{array}{l}\text { " Förderung der Wundheilung } \\
\text { " Infektionen, Ekzeme der Hautfalten (Intertri- } \\
\text { go, Fußpilz) } \\
\text { " Aktinische Keratose } \\
\text { " Basispflege bei Neurodermitis, Psoriasis }\end{array}$ \\
\hline $\begin{array}{l}\text { Bittersüßstängel } \\
\text { Solanum dulcamara L., Dulcamara stipes }\end{array}$ & $\begin{array}{l}\text { Innerliche und äußerliche Anwendung: } \\
\text { " entzündungshemmend } \\
\text { " juckreizlindernd } \\
\text { " Kortison ähnlich } \\
\text { " oberflächenanästhesierend } \\
\text { " adstringierend } \\
\text { " antiallergisch } \\
\text { " antimikrobiell } \\
\text { Innerliche Anwendung: } \\
\text { " stoffwechselanregend }\end{array}$ & $\begin{array}{l}\text { - Chronische Ekzeme } \\
\text { " allergische Dermatitis } \\
\text { " juckende Dermatosen } \\
\text { " Neurodermitis } \\
\text { - Urtikaria } \\
\text { - Milchschorf }\end{array}$ \\
\hline $\begin{array}{l}\text { Johanniskraut } \\
\text { Hypericum perforatum L., Hyperici oleum }\end{array}$ & $\begin{array}{l}\text { Äußerliche Anwendung: } \\
\text { - durchblutungsfördernd } \\
\text { " schmerzstillend } \\
\text { " entzündungshemmend } \\
\text { " fördert die Regeneration der oberen Haut- } \\
\text { schicht } \\
\text { " unterstützt die Hautbarriere } \\
\text { - juckreizlindernd }\end{array}$ & $\begin{array}{l}\text { " schlecht heilende, infizierte Wunden } \\
\text { " als Prophylaxe und Therapie bei Dekubitus } \\
\text { - Verbrennungen } \\
\text { " Neuralgien } \\
\text { " Ekzeme } \\
\text { " Neurodermitis } \\
\text { " Superinfektion m. Staphylokokken }\end{array}$ \\
\hline $\begin{array}{l}\text { Kamille } \\
\text { Matricaria recutita L., Matricariae flos }\end{array}$ & $\begin{array}{l}\text { Äußerliche Anwendung: } \\
\text { - entzündungshemmend } \\
\text { - antibakteriell } \\
\text { - bakterientoxinhemmend } \\
\text { - wundheilungsfördernd } \\
\text { - desodorierend }\end{array}$ & $\begin{array}{l}\text { " Haut- u. Schleimhautentzündungen } \\
\text { " Wundbehandlung auch Dekubitus } \\
\text { " akut nässende Ekzeme } \\
\text { " Spülbehandlung großflächiger Wunden, } \\
\text { Gewebetaschen, Fistelgänge auch infiziert } \\
\text { " Verbrennungen } \\
\text { " Sebostase } \\
\text { " Bakterielle Hauterkrankungen }\end{array}$ \\
\hline
\end{tabular}


- Tab. 2 Therapie von Pruritus, Entzündungsreaktionen und Wunden (Fortsetzung).

\begin{tabular}{|c|c|c|}
\hline Heilpflanze & Wirkung auf die Haut & Indikation \\
\hline $\begin{array}{l}\text { Mahonie } \\
\text { Mahonia aquifolium L., Mahoniae aquifolii } \\
\text { cortex }\end{array}$ & $\begin{array}{l}\text { Innerliche und äußerliche Anwendung: } \\
\text { " entzündungshemmend } \\
\text { " antiproliferativ } \\
\text { " antiseborrhoisch } \\
\text { " antibakteriell } \\
\text { " antimykotisch } \\
\text { " keratolytisch } \\
\text { " juckreizlindernd }\end{array}$ & $\begin{array}{l}\text { - leichte bis mittelschwere Psoriasis } \\
\text { - Seborrhö } \\
\text { " Akne vulgaris } \\
\text { - Dermatosen mit Pruritus }\end{array}$ \\
\hline $\begin{array}{l}\text { Ringelblume } \\
\text { Calendula officinalis L., Calendulae flos }\end{array}$ & $\begin{array}{l}\text { Äußerliche Anwendung: } \\
\text { " wundheilungs- und granulationsfördernd } \\
\text { " entzündungshemmend } \\
\text { " lymphabflussfördernd } \\
\text { " antiödematös } \\
\text { " antibakteriell }\end{array}$ & $\begin{array}{l}\text { - Entzündung von Haut u. Schleimhaut } \\
\text { - Geschwüre } \\
\text { - Ekzeme } \\
\text { - schlecht heilende Wunden } \\
\text { - Dekubitus } \\
\text { - Brustdrüsenentzündungen } \\
\text { - Dermatitis } \\
\text { - Pflege trockener, rissiger Haut }\end{array}$ \\
\hline $\begin{array}{l}\text { Walnussblätter } \\
\text { Juglans regia L., Juglandis folium }\end{array}$ & $\begin{array}{l}\text { Äußerliche Anwendung: } \\
\text { " adstringierend } \\
\text { " antiphlogistisch } \\
\text { " sekretionshemmend } \\
\text { " gewebeverdichtend } \\
\text { " Kapillarpermeabilität hemmend } \\
\text { " juckreizstillend } \\
\text { " oberflächenanästhesierend }\end{array}$ & $\begin{array}{l}\text { Äußerliche Anwendung: } \\
\text { - Ekzeme, leichte } \\
\text { - oberflächliche Entzündungen } \\
\text { - Hyperhidrosis } \\
\text { - Chronische Ekzeme }\end{array}$ \\
\hline $\begin{array}{l}\text { Zaubernuss } \\
\text { Hamamelis virginea L., Hamamelidis foli- } \\
\text { um et cortex }\end{array}$ & $\begin{array}{l}\text { Äußerliche Anwendung: } \\
\text { " adstringierend } \\
\text { " entzündungshemmend } \\
\text { - lokal hämostyptisch und wundheilungsför- } \\
\text { dernd sekretionshemmend } \\
\text { " gewebeverdichtend } \\
\text { - kapillarpermeabilitätshemmend } \\
\text { - juckreizstillend } \\
\text { " wundheilungsfördernd } \\
\text { " vasokonstriktorisch }\end{array}$ & $\begin{array}{l}\text { - zur Wundheilung } \\
\text { - Hautverletzungen } \\
\text { - bei Analfissuren, Hämorrhoiden } \\
\text { - Entzündungen im Genitalbereich } \\
\text { - zur Pflege trockener Altershaut } \\
\text { - Ekzeme } \\
\text { - Neurodermitis } \\
\text { - Dermatitis }\end{array}$ \\
\hline
\end{tabular}

$[1,2,6,7]$

aber nur eine kleine Anzahl von Fallstudien wurde veröffentlicht. Die Wirksamkeit von P. oligandrum gegen drei pathogene Dermatophyten, die bei Haustieren üblich sind, wurde in vitro bestätigt. Im Ergebnis zeigte sich eine starke mykoparasitische Aktivität von P. oligandrum gegen Microsporum canis, Microsporum gypseum und Trichophyton mentagrophytes [5]. Erste Anwendungen zur Hautpilztherapie bei Meerschweinchen und Kaninchen sowie zur unterstützenden Therapie bei Hunden mit fettiger Seborrhoe, zeigen positive Ergebnisse.

In der Zahnmedizin wird er bereits seit längerem zur unterstützenden Parodontaltherapie genutzt, denn er ernährt sich nicht nur von anderen Pilzen, sondern auch von Bakterienproteinen und hier bevorzugt auch von denen anaerober Spezies. Pythium oligandrum nimmt deren Lebensraum ein. Als Stoffwechselprodukt setzt Pythium oligandrum zudem spezielle Enzyme frei, die die Kommunikationswege der Bakterien im bestehenden Biofilm stören. Dadurch fällt es den Erstbesiedlern folgen- den Keimen schwerer, ein funktionierendes System aufzubauen, und gibt den körpereigenen Zellen mehr Zeit, Selbstheilungskräfte auszuüben.

Therapien von Zahnfleischerkrankungen und Entzündungen (z. B. parodontalen Krankheiten wie Mucositis, Parodontitis) profitieren ebenfalls von der Therapie mit Pythium oligandrum [13]. Der Einsatz bei z. B. Hunden mit parodontal geschädigtem Gebiss während und nach Zahnsanierung, bzw. bei Hunden, deren Gesundheitszustand aktuell noch keine Zahnsanierung zulässt, lassen auf positive Ergebnisse hoffen.

Auch in der Tiermedizin werden wir bald unter der Bezeichnung "CleverFungus“ ein eigenes Präparat der Fa. alfavet zur Verfügung haben. 


\section{Zwei Fallbeispiele einfacher \\ Hautaffektionen}

\section{Fall 1}

Signalement

Rasse: EKH, Geschlecht: mk, Alter: unbekannt (Fundkatze Tierheim), Gewicht: 5,1 kg

Vorbericht

Fundkatze wird mit größerer Wunde im Nacken/Schulterbereich vorgestellt ( $\vee$ Abb. $\mathbf{3}$ )

Klinische Untersuchung

Bis auf Wunde obB

Diagnose

Offene, oberflächliche Wunde

\section{Therapie}

- Phlogasept ${ }^{\circledR}$ PlantaVet $10 \%$ ig zur Wundreinigung 3x tgl.

- In den ersten 5 Tagen VulnoPlant ${ }^{\circledR}$ Wundpflege-Spray, bis die Wunde trocken ist, anschließend VulnoPlant ${ }^{\circledR}$ Wundpflege-Creme zur Wundpflege $3 \times$ tgl. bis zum vollständigen Hautschluss

- Halskrause

Verlauf

Wunde heilt beständig ab und ist nach ca. 14 Tagen verheilt.

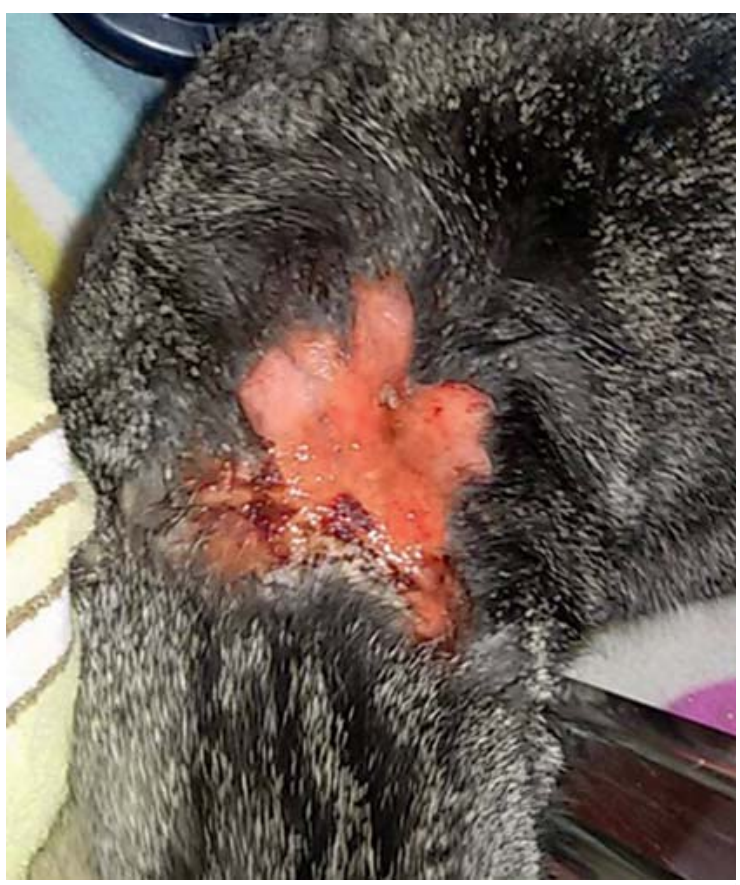

- Abb. 3 Hot Spot bei einer Katze. Ohne Einsatz von Antibiotika. (c) Thoonsen
- Tab. 3 Wirkstoffe der verwendeten Produkte im Vergleich.

\begin{tabular}{|c|c|c|}
\hline $\begin{array}{l}\text { PhlogAsept }{ }^{\circledR} \\
\text { PlantaVet }\end{array}$ & $\begin{array}{l}\text { VulnoPlant }{ }^{\circledR} \text { Wund- } \\
\text { pflegespray }\end{array}$ & $\begin{array}{l}\text { VulnoPlant }{ }^{\circledR} \text { Wund- } \\
\text { pflege-Creme }\end{array}$ \\
\hline $\begin{array}{l}\text { - Kamille } \\
\text { - Salbei } \\
\text { - Hamamelis } \\
\text { - Ringelblume } \\
\text { - Thymol }\end{array}$ & $\begin{array}{l}\text { - Calendula } \\
\text { - Hamamelis } \\
\text { - Salbei } \\
\text { " Thymian }\end{array}$ & $\begin{array}{l}\text { - Johanniskrautöl } \\
\text { - Honig } \\
\text { - Kamille } \\
\text { - Ringelblume } \\
\text { - Hamamelis (Zau- } \\
\text { bernuss) }\end{array}$ \\
\hline
\end{tabular}

Fall 2

Signalement

Rasse: Mischling (Hund aus Griechenland), Geschlecht: wk, Alter: 9 Jahre, Gewicht: 23,8 kg

Vorbericht

- plötzlich nach dem Stallbesuch Blasenbildung auf dem Nasenrücken ( Abb. 4)

- Blasen gehen zeitnah auf

Klinische Untersuchung

Bis auf die vorhandenen Läsionen obB

Diagnose

Dermatitis bullosa unbekannter Genese (Kontaktallergie? Verätzung?)

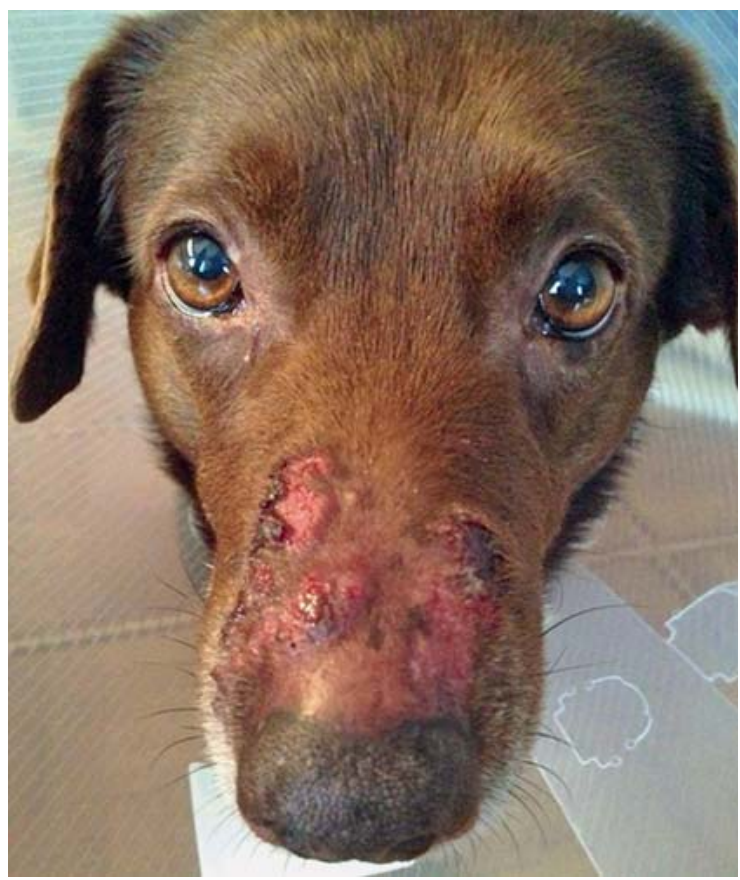

Abb. 4 Dermatitis bullosa. (c) Thoonsen 


\section{Therapie}

- Phlogasept ${ }^{\circledR}$ PlantaVet $10 \%$ ig zur Wundreinigung 2x tgl.

- Ätherisches Öl - Mischung Hund: 3× tgl. dünn auftragen

- Basis Öl: 20 ml Weizenkeimöl; ätherische Öle: 4 Tr. Myrrhe, 4 Tr. Manuka, 4 Tr. Sandelholz, 4 Tr. Lemongras

Verlauf

Die Haut heilt binnen 14 Tagen vollständig und komplikationslos ab.

Autorin

\section{Yvonne Thoonsen}

2000-2007 Studium der Veterinärmedizin an der JustusLiebig-Universität in Gießen. Seit 2008 selbstständig in eigener Praxis in Engelskirchen-Bickenbach. Mitglied der ATF seit 2008 Mitglied der GGTM seit 2010, Mitglied der GPT seit 2015. Ständig aktive Teilnahme in Fort- und Weiterbildungen zu Themen wie z.B. Schmerztherapie, Verhaltenstherapie, Krebstherapie, Osteopathische Akupunktur u.v.m. Seit 2015 in Weiterbildung zur „Biologischen Tiermedizin“, Schwerpunkt Phytotherapie.

\section{Korrespondenzadresse}

Yvonne Thoonsen

Ründerother Straße 27

51766 Engelskirchen

yvonne.thoonsen@t-online.de

\section{Literatur}

[1] Brendieck-Worm, Cäcilia und Melzig, Matthias F. „Phytotherapie in der Tiermedizin“, Thieme Verlag

[2] Bühring, Ursula. „Praxislehrbuch der modernen Pflanzenheilkunde“, Haug Verlag
[3] Gedon, Nathalie K. Y., Müller, Ralf, S. „Neue Entwicklungen bei der Behandlung atopischer Hunde", Kleintier konkret 2018; 21: 3-12; Thieme Verlag

[4] Ley, Martina. „Die Behandlung der felinen atopischen Dermatitis“, Inaugural-Dissertation zur Erlangung der tiermedizinischen Doktorwürde der Tierärztlichen Fakultät der LudwigMaximilians-Universität München 2009

[5] Načeradská, M,; Fafani, M,; Kellnerová, D,; Peková, S,; Girl, P. "Antifungal effects of the biological agent Pythium oligandrum observed in vitro“, PubMed doi:10.1177/ $1098612 \times 16658690$

[6] Reichling, Frater-Schröder, Saller, Fitzi-Rathgen, GachnianMirtscheva. „Heilpflanzenkunde für die Veterinärmedizin“, Spektrum Verlag

[7] Schilcher, H. „Leitfaden Phytotherapie“, Elsevier Verlag

[8] Tretter, Sandra. „Auswirkung von lokal applizierten Fettsäuren auf den epidermalen Wasserverlust, Pruritus und klinischen Hautzustand atopischer und gesunder Hunde“, Inaugural-Dissertation zur Erlangung der tiermedizinischen Doktorwürde der Tierärztlichen Fakultät der Ludwig-Maximilians-Universität München, 2010

[9] Wölfle, Ute; Schempp, Christopf M. „Bitterstoffe - von der traditionellen Verwendung bis zum Einsatz an der Haut“, Zeitschrift für Phytotherapie, Ausgabe 5, Oktober 2018, S. 210 214, Thieme Verlag

[10] https://www.olionatura.de/kosmetikrohstoffe/wirkstoffe/ avocadin-hu-25

[11] https://de.wikipedia.org/wiki/Sphingolipide

[12] https://de.wikipedia.org/wiki/Ceramide

[13] https://www.zantomed.de/wp-content/uploads/2018/01/ Bio-Plus-Probiotika-Pythium-Oligandrum Produktinformation.pdf

\section{Bibliografie}

DOI https://doi.org/10.1055/a-0794-8836

Zeitschrift für Ganzheitliche Tiermedizin 2019; 33: 12-20

(c) Georg Thieme Verlag KG Stuttgart · New York ISSN 0939-7868 